\title{
MicroRNA-212 functions as a tumor-suppressor in human non-small cell lung cancer by targeting SOX4
}

\author{
TINGTING TANG ${ }^{1}$, LITING HUAN ${ }^{2}$, SHUJUAN ZHANG ${ }^{2}$, HUI ZHOU ${ }^{1}$, \\ LEI GU $^{1}$, XIAOHUI CHEN ${ }^{1}$ and LIANG ZHANG ${ }^{1}$ \\ ${ }^{1}$ Department of Hematology and Oncology, The Affiliated Hospital of Hangzhou Normal University, Gongshu, Hangzhou, \\ Zhejiang 310015; ${ }^{2}$ Department of Occupational Medicine, Tai'an Central Hospital Branch, Tai'an, Shandong 271000, P.R. China
}

Received February 24, 2017; Accepted July 21, 2017

DOI: $10.3892 /$ or.2017.5885

\begin{abstract}
Increasing evidence has revealed that aberrant expression of miRNAs contributes to non-small cell lung cancer (NSCLC) development and progression. However, the roles and mechanisms of various miRNAs in NSCLC remain to be determined. In the present study, we confirmed that reduced miR-212 expression was present in NSCLC tissues and cell lines. Our clinical analysis revealed that the reduced miR-212 expression was significantly correlated with poor prognostic features including positive lymph node metastasis and advanced tumor-node-metastasis (TNM) stage. Moreover, we demonstrated that miR-212 is a novel independent prognostic marker for predicting 5-year survival of NSCLC patients. The ectopic overexpression of miR-212 inhibited cell migration, invasion and EMT, while downregulated miR-212 reversed the effect. In addition, miR-212 regulated SOX4 by directly binding to its 3'-untranslated region (3'-UTR), leading to suppression of EMT progression. In clinical samples of NSCLC, miR-212 was negatively correlated with SOX4, which was upregulated in NSCLC. Alteration in SOX4 expression reversed the functional effects of miR-212 in regards to migration, invasion and EMT in the NSCLC cells. In conclusion, our data indicated that miR-212 functions as a tumor-suppressor gene by regulating EMT and metastasis of NSCLC by targeting SOX4 signaling, and may represent a novel potential therapeutic target and prognostic marker for NSCLC.
\end{abstract}

\section{Introduction}

Lung cancer is one of the most common malignancies and the leading cause of cancer-related mortality worldwide. Among all cases of lung cancer, more than $80 \%$ of patients present with non-small cell lung cancer (NSCLC) with a dismal 5-year

Correspondence to: Professor Liang Zhang, Department of Hematology and Oncology, The Affiliated Hospital of Hangzhou Normal University, 126 Wenzhou Road, Gongshu, Hangzhou, Zhejiang 310015, P.R. China

E-mail: zhang18010@126.com

Key words: microRNA-212, lung cancer, SOX4, EMT, invasion survival (1-3). Recently, the high rate of cigarette smoking and environmental pollution have resulted in an increase in the incidence and mortality of lung cancer $(4,5)$. Importantly, despite great improvements in comprehensive diagnosis and medical treatment, the long-term survival of NSCLC patients remains unsatisfactory due to the high rates of recurrence and distant metastasis $(6,7)$. Therefore, it is urgent to elucidate the underlying mechanism responsible for the development and progression of NSCLC and identify novel therapeutic targets involved in NSCLC (8).

MicroRNAs (miRNAs) are a family of endogenous, short single-stranded and non-coding RNAs that negatively modulate gene expression by binding to the 3'-untranslated region (3'-UTR) of target mRNAs to cause degradation or inhibition of translation $(9,10)$. Increasing evidence has confirmed that miRNAs play crucial roles in the physiological and pathological processes in NSCLC including cell differentiation, proliferation, apoptosis, migration and metastasis $(11,12)$. Recently, miR-212, which is located on chromosome 17p13.3, was found to play a critical role in the progression of cancers, including NSCLC (13-16). Previous studies show that miR-212 functions as a regulator of cell invasion, metastasis, proliferation and drug sensitivity. miR-212 performs antimetastatic properties through suppression of SOX4 in breast cancer (17). miR-212 functions as an epigenetic-silenced tumor-suppressor involving in tumor metastasis and invasion of gastric cancer by downregulating PXN expression (18). Moreover, miR-212 inhibits glioblastoma cell proliferation by targeting SGK3 (19). However, the expression of miR-212 is upregulated in pancreatic, esophageal and prostate cancer. miR-212 promotes pancreatic cancer cell growth and invasion by targeting the Hedgehog signaling pathway receptor patched-1 (20). Overexpression of miR-212 predicts a poor prognosis of esophageal cancer patients (21). Thus, the functional significance of miR-212 in cancer initiation, development and process seems to be cancer-type specific. In regards to NSCLC, Li et al reported that miR-212 displays tumor-promoting properties in NSCLC cells and targets the Hedgehog pathway receptor PTCH1 (22); however, Lu et al demonstrated that miR-212 functions as a tumor suppressor in NSCLC by targeting synaptic acetylcholinesterase (23). The expression level of miR-212 and its function in NSCLC are contradictory. Therefore, it is important to identify the roles of miR-212 in NSCLC. 
In the present study, we investigated the expression level and biological function of miR-212 in NSCLC progression. Our data showed that miR-212 was downregulated in NSCLC and the reduced miR-212 was correlated with poor prognostic characteristics and worse 5-year survival of NSCLC patients. We demonstrated that miR-212 regulated the migration and invasion of NSCLC by targeting SOX4 in vitro. Furthermore, miR-212 also suppressed EMT phenotype progression. These data suggest that miR-212 inhibited cell migration and invasion of NSCLC by targeting SOX4. Thus, miR-212 is a novel prognostic biomarker for NSCLC patients.

\section{Materials and methods}

Clinical tissues and cell culture. Clinical specimens were collected from 115 NSCLC patients who received surgical resection in our hospital during 2002 and 2011. All the clinical tissues from the patients were pathologically confirmed as NSCLC before being used for further experiments in the present study. Informed consent was obtained from every patient involved in the present study. Approval for experiments involving patient samples was obtained from the Institutional Research Ethics Committee of The Affiliated Hospital of Hangzhou Normal University.

Cell lines including H292, H1299, A549, SPC-A1 and BEAS-2B were purchased from the Cell Bank of Chinese Academy of Sciences (Shanghai, China) and the American Type Culture Collection (ATCC; Rockville, MD, USA). All cells were cultured in Dulbecco's modified Eagle's medium (DMEM) supplemented with 10\% fetal bovine serum (FBS) (both from Gibco Co., New York, NY, USA). Cell cultures were kept in cell incubators with humidified atmosphere and $5 \% \mathrm{CO}_{2}$ at $37^{\circ} \mathrm{C}$.

Quantitative real-time reverse transcription-PCR ( $q R T-P C R)$. RNeasy Mini kit (Qiagen, Inc., Valencia, CA, USA) was used to extract RNA from the NSCLC tissues and cells. Transcriptional First Strand cDNA Synthesis kit (Roche, Indianapolis, IN, USA) and SYBR-Green PCR Master Mix (Applied Biosystems, Foster City, CA, USA) were used for reverse transcription reactions and real-time PCR. Primers for miR-212, U6, SOX4 and GAPDH were purchased from GeneCopoeia (Guangzhou, China). U6 and GAPDH were the internal controls for miR-212 and SOX4, respectively.

Western blotting. Total protein was extracted in RIPA buffer (Beyotime, Shanghai, China) containing a protease and phosphatase inhibitor, and then the protein concentration was determined using the BCA Protein Assay kit (both from Thermo Scientific, Rockford, IL, USA). Equal protein $30 \mu \mathrm{g}$ was loaded and separated by $10 \%$ SDS-PAGE and transferred to polyvinyllidene diflouride (PVDF) membranes (Millipore, Billerica, MA, USA). The membranes were incubated with respective primary antibodies: SOX4 and GAPDH $(1: 1,000$; Cell Signaling Technology, Inc., Danvers, MA, USA) at $4^{\circ} \mathrm{C}$ overnight after blocking with $5 \%$ non-fat milk in TBS. Then, the membranes were washed three times with Tris-buffered saline with Tween-20 (TBST) and incubated with appropriate peroxidase-conjugated secondary antibody for $2 \mathrm{~h}$ at room temperature (ZSGB-BIO, Beijing, China). Protein bands were visualized using an enhanced chemiluminescence kit (Amersham, Little Chalfont, UK).

Immunohistochemical analysis. The tissues were fixed in formalin, embedded with paraffin and sliced into $4-\mu \mathrm{m}$ sections for immunohistochemical staining. After deparaffinizing, dehydrating and antigen retrieval, the slides were incubated in $3 \% \mathrm{H}_{2} \mathrm{O}_{2}$ for blocking endogenous peroxidase activity. Then, after incubating with $10 \%$ goat serum for 30 min, E-cadherin and vimentin (1:300; Cell Signaling Technology, Inc.) antibodies were applied as the primary antibodies by a streptavidin peroxidase-conjugated (SP-IHC) method. The percentage of positive cells was expressed as: 0 for $<10 \%$; 1 for $10-30 \%$; 2 for $31-50 \%$; 3 for $>50 \%$.

Cell transfection. miR-212 mimic and miR-212 inhibitor were obtained from GeneCopoeia Inc. SOX4 expression vector and SOX4-specific siRNA were purchased from Ruibo Biotechnology Corp. (Guangzhou, China). The tranfection of these vectors into NSCLC cells was performed in 6-well plates using Lipofectamine 2000 (Invitrogen, Carlsbad, CA, USA) based on the instructions from the manufacturer.

Transwell assays. The migration and invasion abilities of the NSCLC cells were investigated by Transwell assays without or with Matrigel. Generally, NSCLC suspended in basal DMEM was seeded into the upper chamber and the lower chambers contained $600 \mu \mathrm{l}$ DMEM with $20 \%$ FBS. Twenty-four to $48 \mathrm{~h}$ later, NSCLC cells that had migrated or invaded through the membranes and stayed on the lower surface were stained with crystal violet. Cell number for the migrated or invaded cells was counted under a microscope.

Luciferase reporter assay. Wild-type SOX4 3'-UTR sequence and the mutated SOX4 3'-UTR sequence were constructed into the pGL3 control vector (Promega, Madison, WI, USA) to obtain wt SOX4-3'-UTR and mt SOX4-3'-UTR vectors, respectively. For the luciferase reporter assay, NSCLC cells were co-transfected with the wild-type construct or mutant construct, and, miR-212 mimics or inhibitor or control or negative control vector. Forty-eight hours after transfection, cells were harvested and lysed. The Dual-Luciferase Reporter Assay System (Promega, Shanghai, China) was used to determine firefly and Renilla luciferase activities.

Statistical analysis. Data are presented as the mean \pm SD and at least three independent replicates were performed. SPSS software 16.0 (SPSS, Inc., Chicago, IL, USA) and GraphPad Prism 6.0 (GraphPad Software, Inc., La Jolla, CA, USA) were used with a two-tailed Student's t-test, Pearson's correlation analysis, Kaplan-Meier method and the log-rank test to evaluate the statistical significance. Significant differences were defined as $\mathrm{P}<0.05$.

\section{Results}

miR-212 expression is downregulated in NSCLC tissues and cell lines. To determine the expression level of miR-212 in NSCLC, we first evaluated the expression of miR-212 in 115 paired NSCLC and adjacent normal lung tissues. miR-212 
A

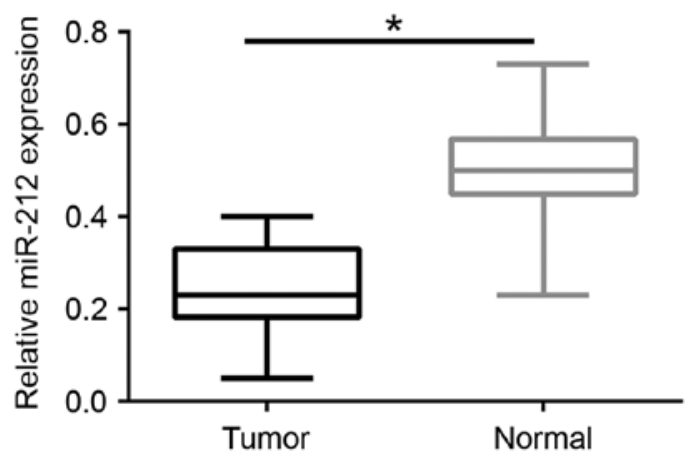

B

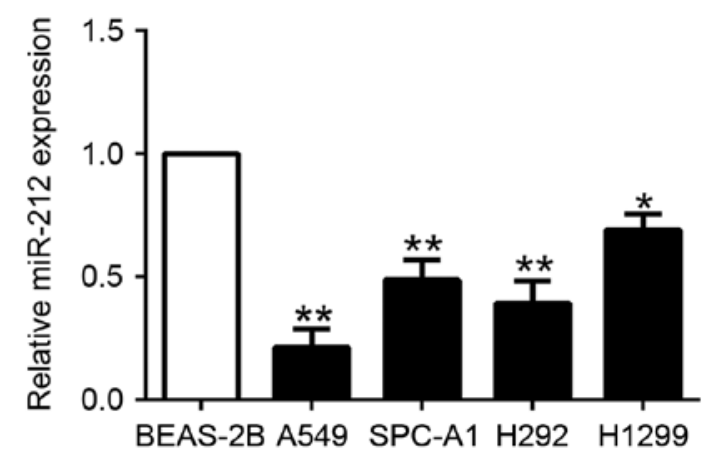

Figure 1. Expression of miR-212 in NSCLC tissues and cell lines. (A) Quantification of the data revealed that the mean level of miR-212 expression in NSCLC tissues was significantly lower than that in matched adjacent non-cancer tissues. (B) Comparing differences in the expression levels of miR-212 between NSCLC cell lines compared to the normal gastric epithelial cell line (BEAS-2B). $n=6$ repeats with similar results. U6 snRNA was used as internal control; ${ }^{*} \mathrm{P}<0.05,{ }^{* *} \mathrm{P}<0.01$.

Table I. Correlation between miR-212 expression and clinicopathological features of the NSCLC cases $(n=115)$.

\begin{tabular}{|c|c|c|c|c|}
\hline \multirow[b]{2}{*}{$\begin{array}{l}\text { Clinical } \\
\text { parameters }\end{array}$} & \multirow[b]{2}{*}{$\begin{array}{l}\text { Cases } \\
\text { (n) }\end{array}$} & \multicolumn{2}{|c|}{ Expression level } & \multirow[b]{2}{*}{ P-value } \\
\hline & & $\begin{array}{c}\operatorname{miR}-212^{\text {high }} \\
\quad(n=54)\end{array}$ & $\begin{array}{c}\mathrm{miR}-212^{\text {low }} \\
\quad(\mathrm{n}=61)\end{array}$ & \\
\hline Age (years) & & & & 0.716 \\
\hline$<60$ & 36 & 16 & 20 & \\
\hline$\geq 60$ & 79 & 38 & 41 & \\
\hline Sex & & & & 0.589 \\
\hline Male & 89 & 43 & 46 & \\
\hline Female & 26 & 11 & 15 & \\
\hline Histologic type & & & & 0.724 \\
\hline Squamous & 68 & 31 & 37 & \\
\hline Adenocarcinoma & 47 & 23 & 24 & \\
\hline $\begin{array}{l}\text { Lymph node } \\
\text { metastasis }\end{array}$ & & & & $0.001^{\mathrm{a}}$ \\
\hline Negative & 83 & 47 & 36 & \\
\hline Positive & 32 & 7 & 25 & \\
\hline TNM stage & & & & $0.003^{\mathrm{a}}$ \\
\hline $\mathrm{I}+\mathrm{II}$ & 78 & 44 & 34 & \\
\hline III+IV & 37 & 10 & 27 & \\
\hline
\end{tabular}

${ }^{\mathrm{a} P}<0.05$. NSCLC, non-small cell lung cancer; TNM, tumor-nodemetastasis.

was significantly decreased in the NSCLC tissues compared to that noted in the matched tumor-adjacent tissues $(\mathrm{P}<0.05$; Fig. 1A). Similarly, miR-212 was markedly downregulated in a panel of NSCLC cells (A549, SPC-A1, H292 and H1299) as compared to that observed in the normal lung epithelial cell line BEAS-2B $(\mathrm{P}<0.05$; Fig. 1B). These data identified miR-212 as a tumor-suppressor of the progrssion of NSCLC.

Correlations between miR-212 expression and clinical characteristics of the NSCLC cases. To investigate the clinical significance of reduced miR-212 in NSCLC, we divided the

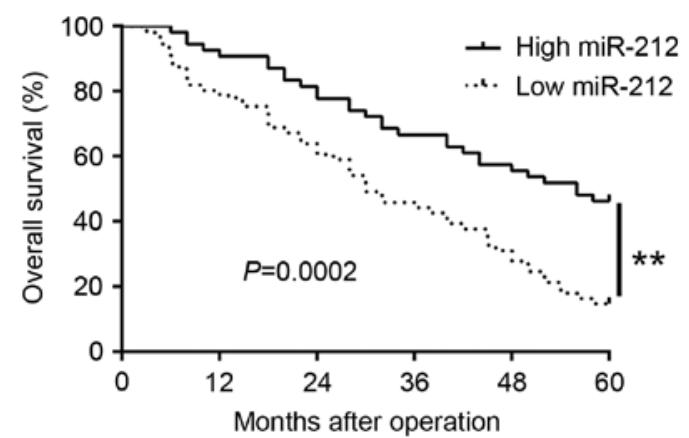

Figure 2. The prognostic value of miR-212 for NSCLC patients. NSCLC patients with higher expression of miR-212 had better overall survival; ${ }^{* *} \mathrm{P}<0.01$.

patients into two different miR-212 groups according to the median expression level. As shown in Table I, decreased miR-212 expression was significantly correlated with advanced tumor-node-metastasis $(\mathrm{TNM})$ stage $(\mathrm{P}=0.003)$ and positive lymph node metastasis $(\mathrm{P}=0.001)$. These results suggest that the reduced miR-212 was associated with poor prognostic features of NSCLC. Furthermore, Kaplan-Meier analysis revealed that the patients with low miR-212 expression had obviously shorter overall survival than those with high miR-212 expression ( $\mathrm{P}=0.0002$; Fig. 2). In addition, miR-212 expression was an independent factor for predicting 5 -year overall survival in NSCLC patients $(\mathrm{P}=0.001,0.001$, respectively; Table II). Taken together, these data indicate that miR-212 is a potential biomarker for predicting the outcome of NSCLC patients.

miR-212 inhibits NSCLC cell migration and invasion in vitro. To investigate the potential role of miR-212 in NSCLC, we performed gain- and loss-of-function experiments based on miR-212 mimics and inhibitors, which were transfected into A549 (P<0.05; Fig. 3A) and H1299 cells ( $<<0.05$; Fig. 3C), respectively. As measured by Matrigel-uncoated (for migration assay) and Matrigel-coated (for invasion assay) Transwell assays, ectopic expression of miR-212 in A549 cells resulted in a significant reduction of cell migration and invasion capacity ( $\mathrm{P}<0.05$; Fig. 3B). In contrast, transfection of H1299 cells 
Table II. Univariate and multivariate analyses of prognostic factors in NSCLC patients.

\begin{tabular}{|c|c|c|c|c|c|c|}
\hline \multirow[b]{2}{*}{ Variables } & \multicolumn{3}{|c|}{ Univariate analysis } & \multicolumn{3}{|c|}{ Multivariate analysis } \\
\hline & HR & $95 \%$ CI & P-value & HR & $95 \%$ CI & P-value \\
\hline TNM stage & 2.013 & $1.409-2.872$ & $0.002^{\mathrm{a}}$ & 1.862 & $1.283-2.708$ & $0.003^{\mathrm{a}}$ \\
\hline Lymph node metastasis & 2.798 & $1.879-5.682$ & $0.001^{\mathrm{a}}$ & 2.214 & $1.387-3.263$ & $0.002^{\mathrm{a}}$ \\
\hline miR-212 & 3.967 & $1.583-7.382$ & $0.001^{\mathrm{a}}$ & 3.223 & $1.237-6.962$ & $0.001^{\mathrm{a}}$ \\
\hline
\end{tabular}

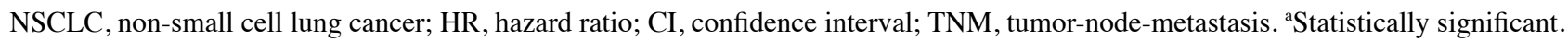
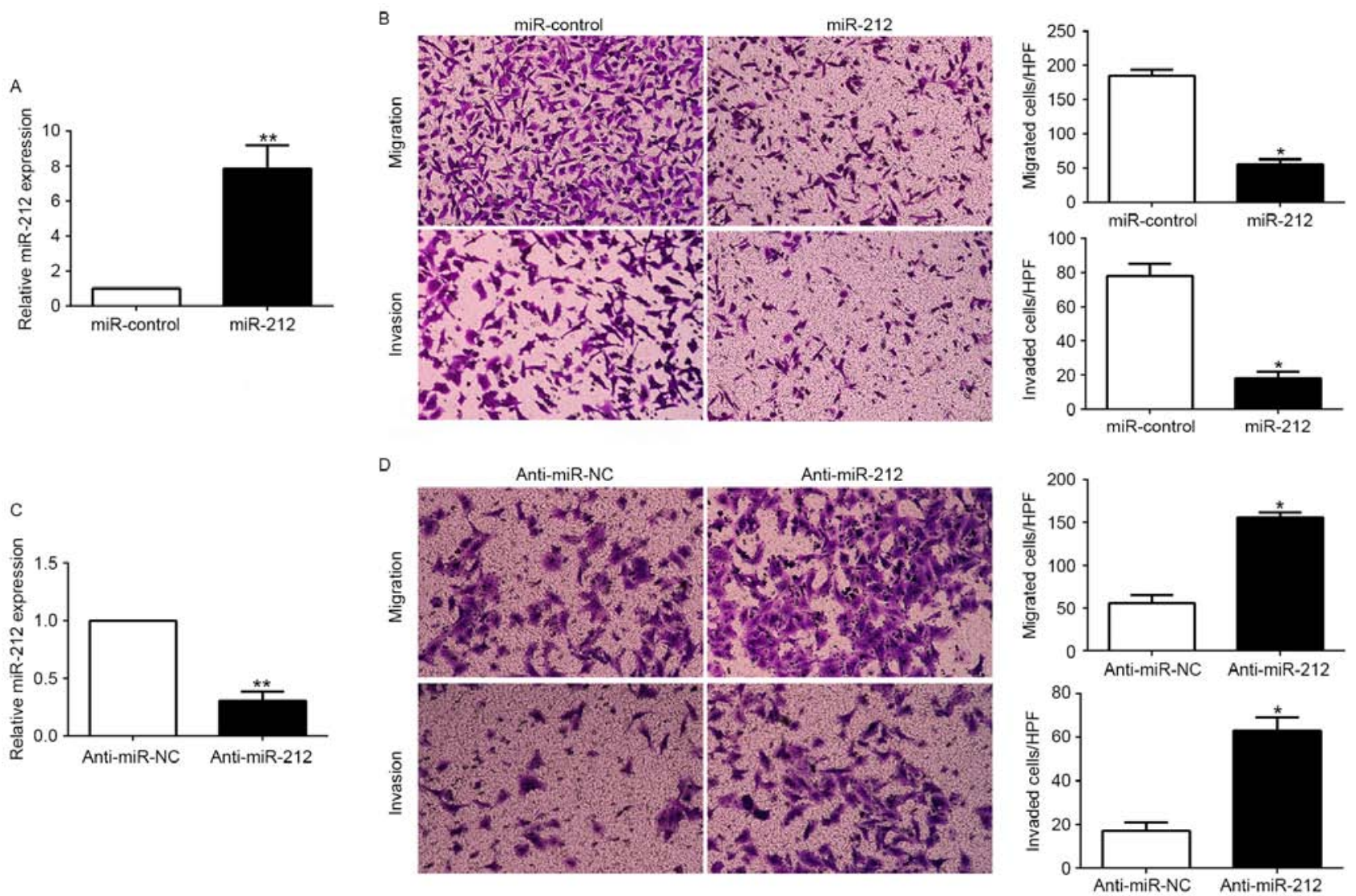

Figure 3. miR-212 inhibits NSCLC cell migration and invasion in vitro. (A) A549 cells that were transfected with the corresponding miRNA vectors were subjected to qRT-PCR for miR-212. (B) Cell migration and invasion abilities were inhibited by overexpression of miR-212 in the A549 cells as measured by Transwell assays. (C) H1299 cells that were transfected with miR-212 inhibitors (anti-miR-212) and a negative control were subjected to qRT-PCR for miR-212 (D) Cell migration and invasion abilities were increased by knockdown of miR-212 in the H1299 cells as measured by Transwell assays. $\mathrm{n}=6$ independent experiments; ${ }^{*} \mathrm{P}<0.05,{ }^{* *} \mathrm{P}<0.01$.

with miR-212 inhibitor obviously increased cell migration and invasion $(\mathrm{P}<0.05$; Fig. 3D). These data demonstrated that miR-212 suppressed the migration and invasion of NSCLC cells in vitro.

miR-212 suppresses epithelial-to-mesenchymal transition in NSCLC cells. EMT has been identified as having a crucial role in the induction of metastatic progression of cancer (24). To investigate the potential role of miR-212 in modulating NSCLC metastasis, EMT markers were assessed. We found that miR-212 overexpression increased the epithelial marker $\mathrm{E}$-cadherin and inhibited $\mathrm{N}$-cadherin and vimentin expression $(\mathrm{P}<0.05$; Fig. 4A). In contrast, miR-212 knockdown decreased E-cadherin expression and increased $\mathrm{N}$-cadherin and vimentin expression ( $\mathrm{P}<0.05$; Fig. 4B). In addition, we further explored the correlation between miR-212 expression and EMT markers in NSCLC tissues. We found that the E-cadherin expression in the high miR-212 group was higher than that in the low miR-212 group. Conversely, the expression level of vimentin in the high miR-212 group was markedly lower than that in the low miR-212 group ( $\mathrm{P}<0.05$; Fig. 4C). Taken together, these results suggest that miR-212 functions as a suppressor of EMT in NSCLC cells.

SOX4 is a direct target of miR-212. To explore the molecular mechanism by which miR-212 suppressed NSCLC 

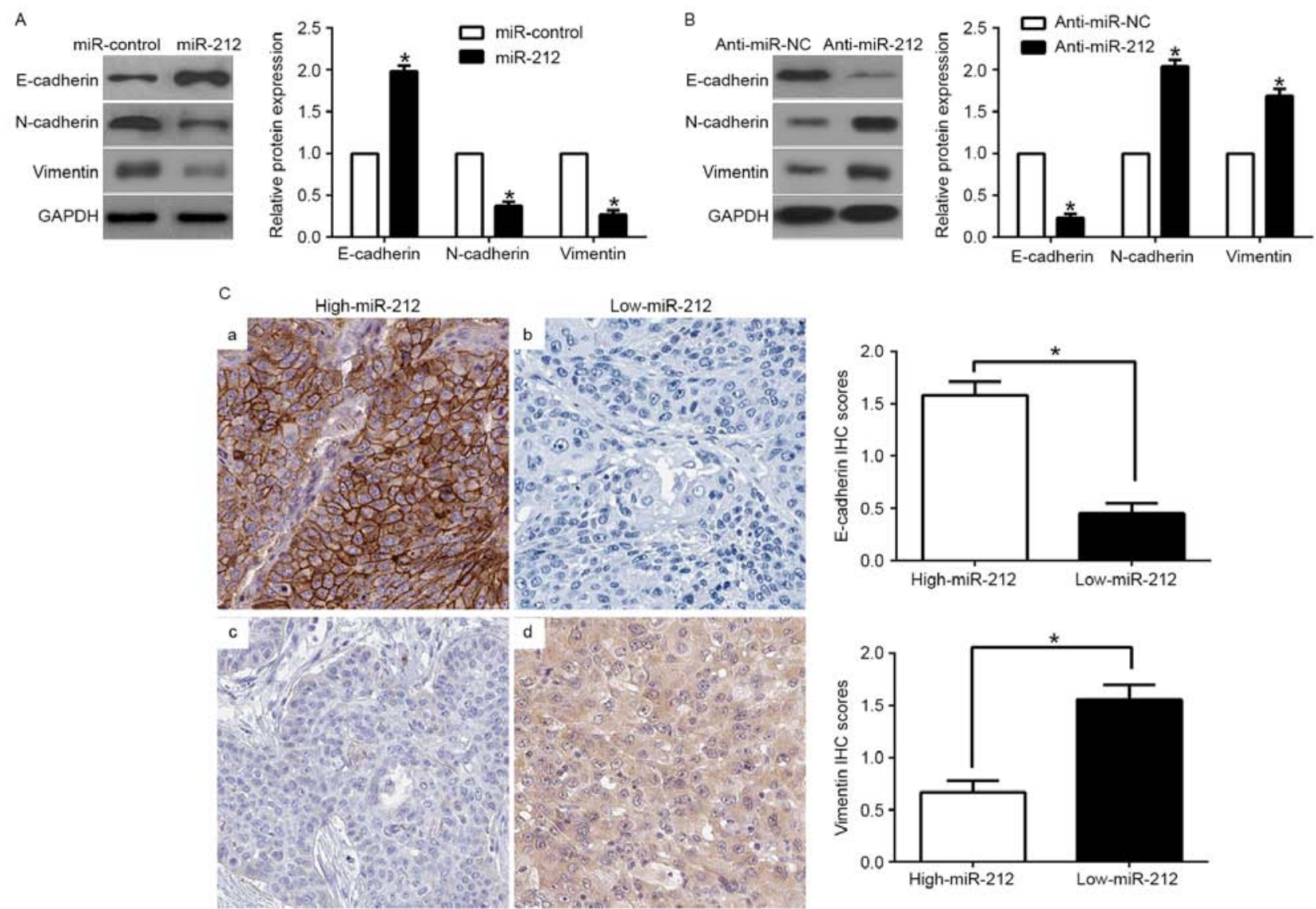

Figure 4. miR-212 suppresses epithelial-to-mesenchymal transition in NSCLC cells. (A) Overexpression of miR-212 in A549 cells increased the expression of the epithelial cell marker E-cadherin and decreased the expression of the mesenchymal cell markers N-cadherin and vimentin. (B) In contrast, the expression of the antagomiR to miR-212 decreased E-cadherin expression and increased $\mathrm{N}$-cadherin and vimentin expression. Immunohistochemical analysis of E-cadherin and vimentin in NSCLC samples. (C) In cases of high miR-212 expression (a and c); there was strong E-cadherin and no detectable vimentin protein expression in the same tissue section. In contrast, in the case of low miR-212 expression (b and d), there was no detectable E-cadherin and strong vimentin protein expression. Values are depicted as mean $\pm \mathrm{SEM} ;{ }^{*} \mathrm{P}<0.05$ by t-test.

migration and invasion, we used public bioinformatic algorithms (TargetScan 6.2 and MiRanda) to search for candidate target genes. We selected sex-determining region Y-box 4 (SOX4) for proceeding with experimental confirmation, which was confirmed in other cancers. The 3'-UTR of SOX4 mRNA contains a putative complementary site of miR-212 (Fig. 5A). To confirm the prediction, we constructed the wild-type (WT) and mutant (MUT) 3'-UTR of SOX4 plasmids. Luciferase reporter assays revealed that ectopic miR-212 expression significantly inhibited the luciferase activity of SOX4 containing a WT 3'-UTR, but did not suppress the activity of SOX4 with a MUT 3'-UTR; in contrary, miR-212 inhibition increased the luciferase activity of wild-type of 3'-UTR of SOX4 while had no effect on the mutant SOX4 3'-UTR (P<0.01; Fig. 5B). Moreover, we demonstrated that miR-212 overexpression significantly inhibited SOX4 mRNA and protein expression in A549 cells, whereas the downregulation of miR-212 prominently increased the mRNA and protein levels of SOX4 in H1299 cells ( $\mathrm{P}<0.05$, respectively; Fig. 5C and D).

Subsequently, we examined the correlation between miR-212 expression and SOX4 in NSCLC tissues. We found that the expression of SOX4 mRNA and protein in the miR-212 high-expressing tumors were significantly lower than those in the miR-212 low-expressing tumors $(\mathrm{P}<0.05$, respectively;
Fig. 5E and F). Taken together, these results suggest that miR-212 inhibits SOX4 expression by direct binding to the 3'-UTR of SOX4.

Alteration in SOX4 expression reverses the functional effects of miR-212 in NSCLC cells. To clarify that SOX4 is a functional target of miR-212, SOX4 was restored by a plasmid vector in miR-212-overexpressing A549 cells ( $\mathrm{P}<0.05$; Fig. 6A). Furthermore, SOX4 overexpression increased cell migration, invasion $(\mathrm{P}<0.05$, respectively; Fig. $6 \mathrm{~B})$ and promoted EMT $(\mathrm{P}<0.05$; Fig. 6E). Similarly, SOX4 knockdown by a specific siRNA in miR-212-suppressive $\mathrm{H} 1299$ cells $(\mathrm{P}<0.05$; Fig. 6C) significantly inhibited cell migration, invasion $(\mathrm{P}<0.05$, respectively; Fig. 6D) and EMT $(\mathrm{P}<0.05$; Fig. 6F $)$. These data demonstrated that SOX4 is a downstream mediator in the function of miR-212 in NSCLC.

\section{Discussion}

Increasing evidence has confirmed that aberrant miRNAs play a critical role in NSCLC development and progression. Previous studies indicated that miR-212 is involved in the pathogenic process of diverse human cancers. miR-212 downregulates SMAD2 expression to suppress the G1/S phase 
A

WT-SOX4-3'-UTR 3'-...GCUAUUCAGCAUACGACGAAUGA...-5'

Hsa-miR-212 5'-ACCUUGGCUCUAGACUGCUUACU-3

MT-SOX4-3'-UTR 3'-...GCUAUUCAGCAUACGACCCCUGA...-5'

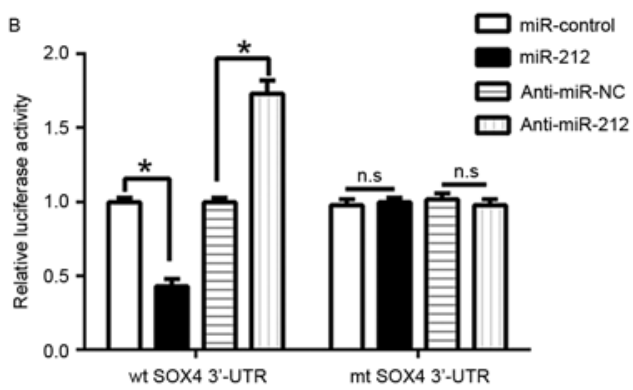

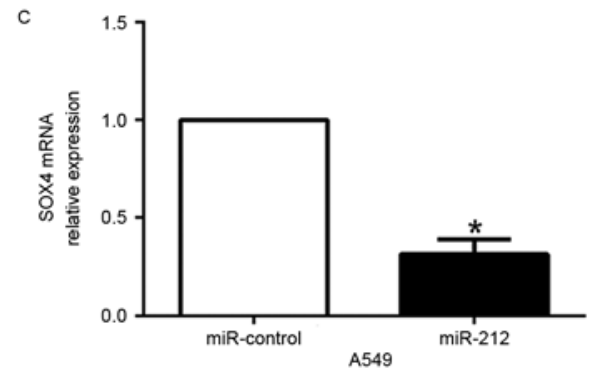
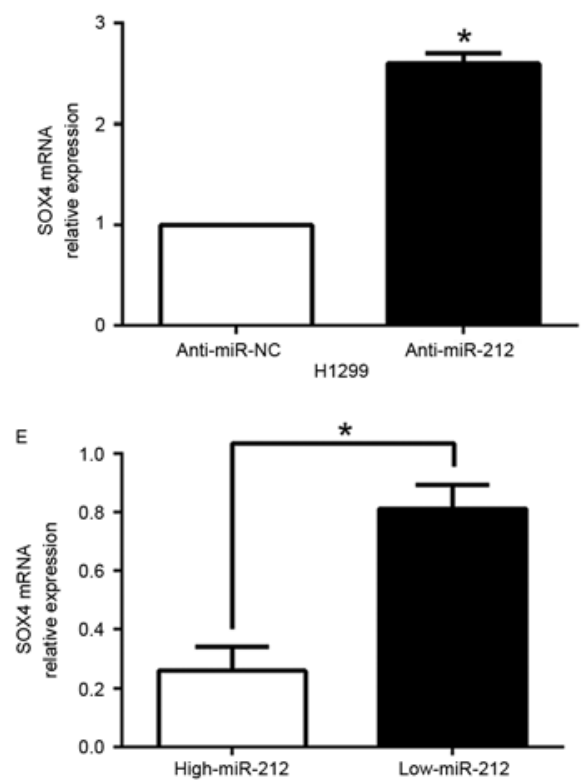

D
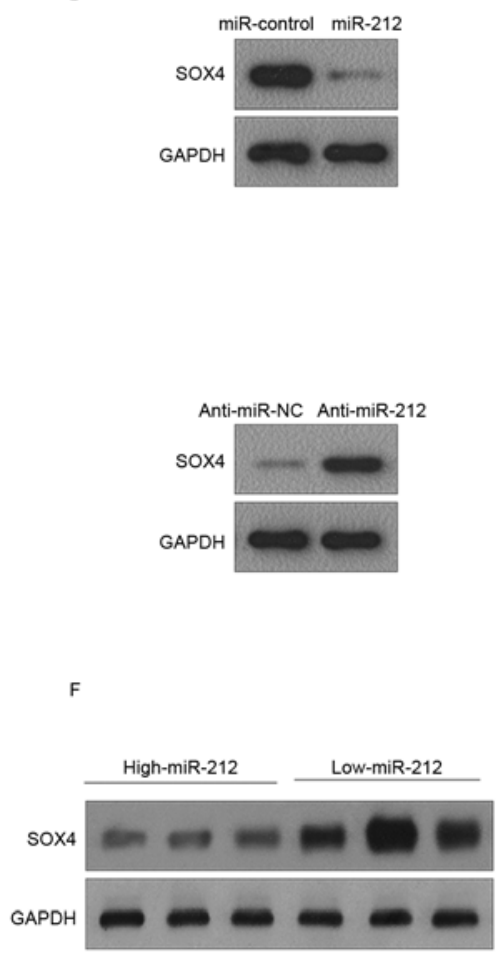
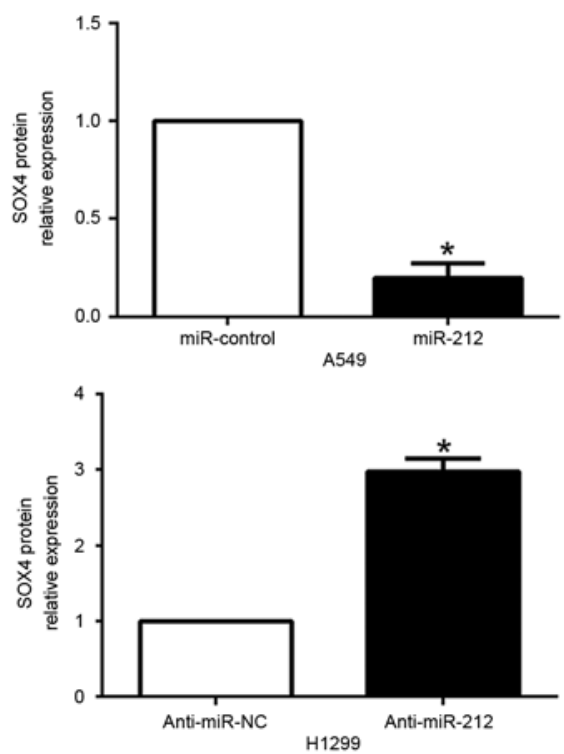

*

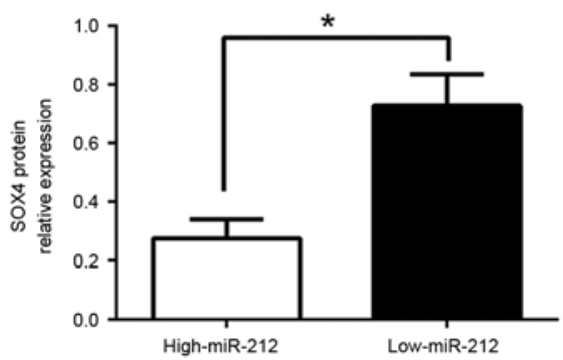

Figure 5. SOX4 is a direct target of miR-212 in NSCLC. (A) miR-212 and its putative binding sequence in the 3'-UTR of SOX4. The mutant binding site was generated in the complementary site for the seed region of miR-212. (B) miR-212 significantly suppressed the luciferase activity that carried the wild-type (WT), but not the mutant (MUT) 3'-UTR of SOX4. (C) qRT-PCR analysis of SOX4 mRNA expression in A549 cells transfected with the miR-212 or miRcontrol vector and H1299 cells transfected with the anti-miR-212 or anti-miR-NC vector. (D) Overexpression of miR-212 reduced the expression of SOX4 protein in A549 cells and knockdown of miR-212 increased the level of SOX4 protein in H1299 cells. (E) The expression of SOX4 mRNA in miR-212 highexpressing tumors was significantly lower than that in miR-212 low-expressing tumors. (F) The expression of SOX4 protein in miR-212 high-expressing tumors was significantly lower than that in miR-212 low-expressing tumors. $\mathrm{n}=6$ repeats with similar results, ${ }^{\prime} \mathrm{P}<0.05$; n.s. not significant

transition of the cell cycle and EMT in cervical cancer cells (25). miR-212 is downregulated and suppresses methyl$\mathrm{CpG}$-binding protein $\mathrm{MeCP} 2$ in gastric cancer (26). Moreover, regulation of heparin-binding EGF-like growth factor by miR-212 was found to be a potential mechanism for the acquired cetuximab-resistance in head and neck squamous cell carcinoma (27). miR-212 was found to inhibit hepatocellular carcinoma cell proliferation and induce apoptosis by targeting FOXA1 (28). In contrast, overexpression of miR-212 was found to be associated with poor prognosis of patients with pancreatic ductal adenocarcinoma (29). In the present study, we demonstrated that miR-212 expression was significantly downregulated in NSCLC tissues and cell lines. These data indicate that miR-212 may be involved in the pathogenesis of NSCLC as a tumor suppressor. Following clinical analysis of miR-212 with pathological characteristics, we found that reduced miR-212 was obviously associated with advanced TNM stage and positive lymph node metastasis. Moreover, our data indicated that high expression of miR-212 predicated a significant better 5-year survival for NSCLC patients. Multivariate Cox repression analysis indicated that miR-212 was an independent prognostic factor for predicting the survival of NSCLC patients. Taken together, these results indicate that miR-212 expression is pivotal for the prognostic 

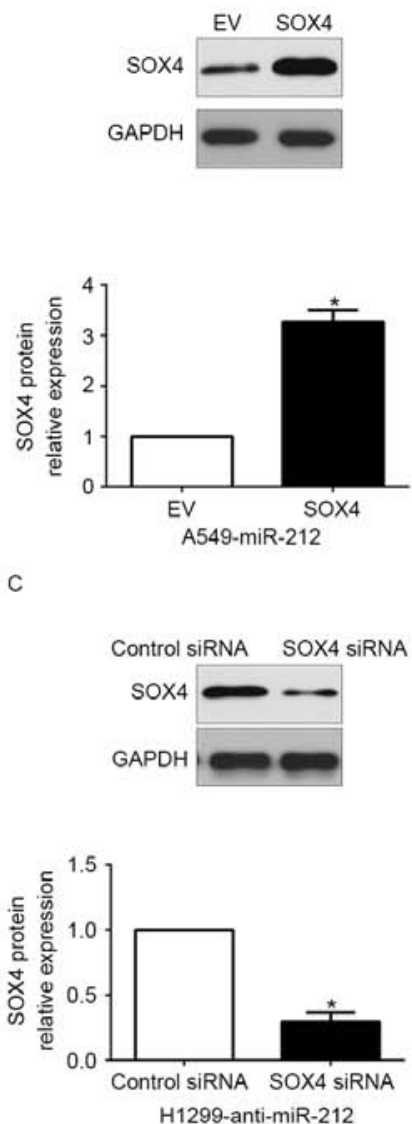

E

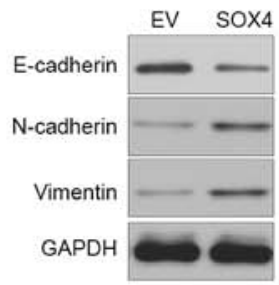

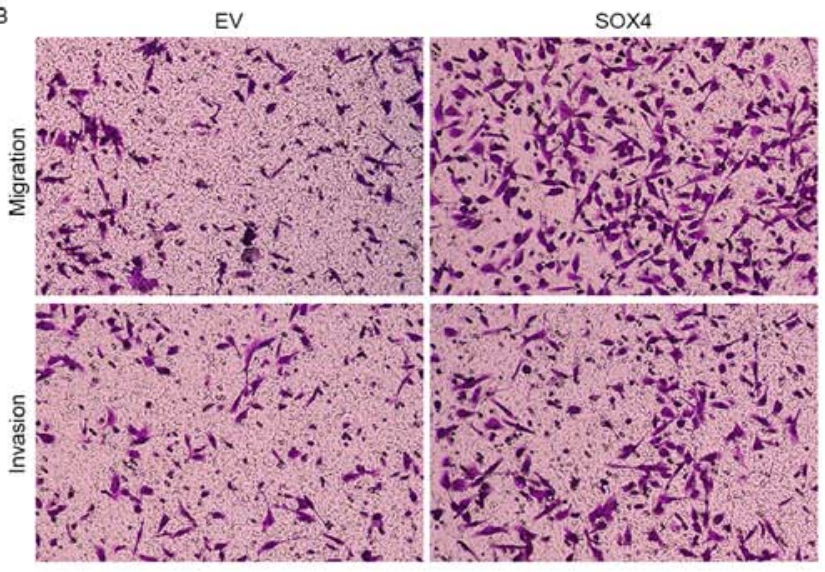

D
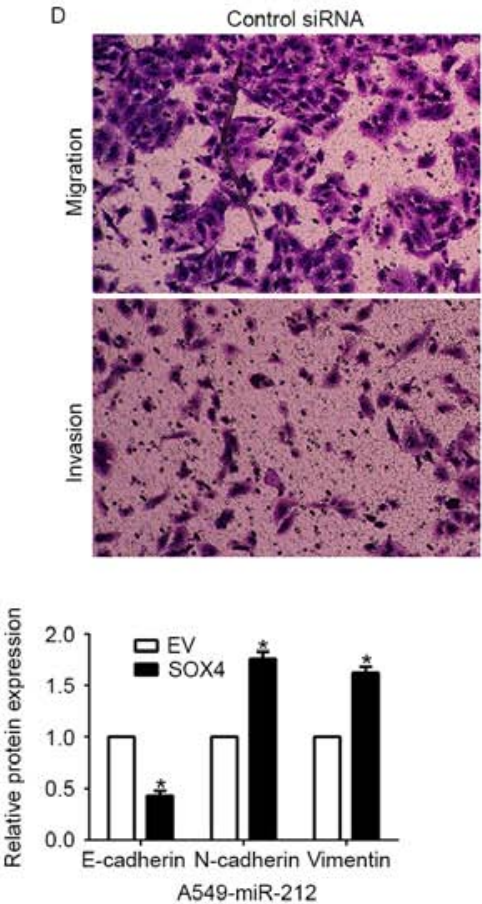
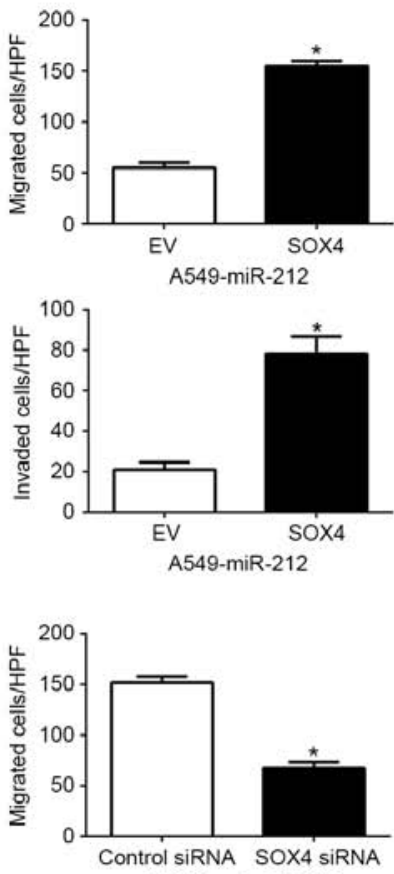

H1299-anti-miR-212

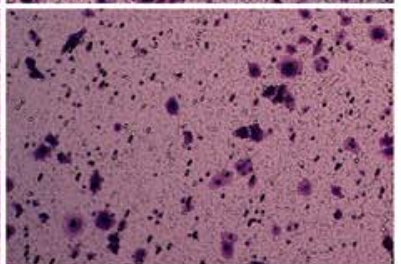

F Control siRNA SOX4 siRNA
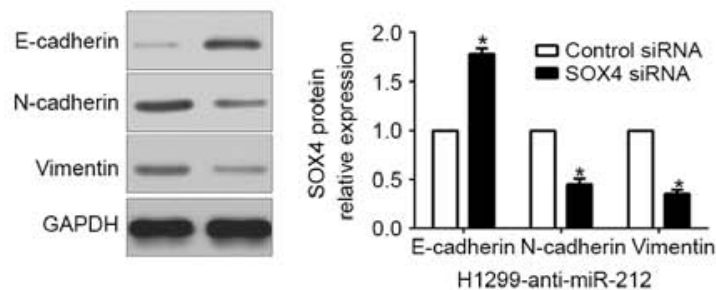

Figure 6. Alterations of SOX4 partially reverse miR-212-mediated NSCLC cell migration, invasion and EMT. (A) miR-212-overexpressing A549 cells that were transfected with EV or SOX4 expression plasmid were subjected to western blot analysis for SOX4. (B) Cell migration and invasion of the miR-212-overexpressing A549 cells was increased by SOX4 overexpression. (C) miR-212-silenced H1299 cells that were transfected with scrambled siRNA or SOX4 siRNA were subjected to western blot analysis for SOX4. (D) SOX4 knockdown abrogated the effects of miR-212 knockdown on H1299 cells. (E) Western blot analysis of EMT marker proteins in A549 cells stably expressing miR-212 transduced with SOX4 or control vector. (F) Western blot analysis of indicated proteins in H1299 cells stably expressing miR-212 inhibitor transfected with SOX4 siRNA or control siRNA. n=6 independent experiments. EV, empty vector; ${ }^{*} \mathrm{P}<0.05$.

outcome in NSCLC patients. Functionally, we found that miR-212 overexpression reduced while miR-212 knockdown increased SOX4 expression in NSCLC cell lines by directly interacting with the 3'-UTR of SOX4 mRNA. In addition, we found the SOX4 mRNA and protein expression was inversely correlated with miR-212 expression in NSCLC tissues. In addition, alteration of SOX4 expression partially reversed miR-212-induced function in NSCLC cells. Taken together, SOX4 was identified as a functional target gene of miR-212 in NSCLC.

Sex-determining region Y-related high-mobility group box 4 (SOX4) has been reported to be upregulated in multiple cancers, including NSCLC (30). SOX4 is involved in early embryogenesis and cell phenotype decisions. Previous studies confirmed that SOX4 is a critical component of the PTEN/
PI3K/AKT pathway in prostate cancer (31). SOX4 is a frequent target of retroviral insertional mutagenesis and contributes to transformation in murine hematopoietic cells. In the present study, we found that miR-212 inhibited tumor migration and invasion and the EMT phenotype by targeting SOX4 in vitro. Furthermore, we found that miR-212 expression was inversely correlated with EMT marker (E-cadherin and vimentin) expression, which reinforce the biological function of miR-212 on EMT. These data confirmed that the functional effect of miR-212 in NSCLC in vitro was dependent on SOX4.

In summary, we demonstrated that miR-212 was downregulated in NSCLC tissues and cell lines, and its expression was correlated with malignant clinicopathological features. Furthermore, we confirmed that overexpression of miR-212 inhibited cell migration and invasion in vitro by inhibiting 
SOX4-mediated EMT signaling pathway. These results suggest that miR-212 is a potential invasion-associated tumor suppressor in NSCLC. In the future, therapeutic interventions concentrating on miR-212-SOX4 may help suppress the development and metastasis of NSCLC.

\section{References}

1. Siegel RL, Miller KD and Jemal A: Cancer statistics, 2015. CA Cancer J Clin 65: 5-29, 2015.

2. Zornosa C, Vandergrift JL, Kalemkerian GP, Ettinger DS, Rabin MS, Reid M, Otterson GA, Koczywas M, D'Amico TA, Niland JC, et al: First-line systemic therapy practice patterns and concordance with NCCN guidelines for patients diagnosed with metastatic NSCLC treated at NCCN institutions. J Natl Compr Canc Netw 10: 847-856, 2012.

3. Goldstraw P, Ball D, Jett JR, Le Chevalier T, Lim E, Nicholson AG and Shepherd FA: Non-small-cell lung cancer. Lancet 378: $1727-1740,2011$.

4. Blais $\mathrm{N}$ and Kassouf E: Maintenance therapies for non-small cell lung cancer. Front Oncol 4: 213, 2014.

5. Chen W, Zheng R, Zeng H, Zhang S and He J: Annual report on status of cancer in China, 2011. Chin J Cancer Res 27: 2-12, 2015.

6. Chen Z, Fillmore CM, Hammerman PS, Kim CF and Wong KK: Non-small-cell lung cancers: A heterogeneous set of diseases. Nat Rev Cancer 14: 535-546, 2014.

7. Lemjabbar-Alaoui H, Hassan OU, Yang YW and Buchanan P: Lung cancer: Biology and treatment options. Biochim Biophys Acta 1856: 189-210, 2015.

8. Ferlay J, Shin HR, Bray F, Forman D, Mathers C and Parkin DM: Estimates of worldwide burden of cancer in 2008: GLOBOCAN 2008. Int J Cancer 127: 2893-2917, 2010.

9. Kim VN: MicroRNA biogenesis: Coordinated cropping and dicing. Nat Rev Mol Cell Biol 6: 376-385, 2005.

10. Shivdasani RA: MicroRNAs: Regulators of gene expression and cell differentiation. Blood 108: 3646-3653, 2006.

11. Cao Q, Mao ZD, Shi YJ, Chen Y, Sun Y, Zhang Q, Song L and Peng LP: MicroRNA-7 inhibits cell proliferation, migration and invasion in human non-small cell lung cancer cells by targeting FAK through ERK/MAPK signaling pathway. Oncotarget 7 : 77468-77481, 2016.

12. Zhu X, Li Y, Shen H, Li H, Long L, Hui L and Xu W: miR-137 inhibits the proliferation of lung cancer cells by targeting Cdc42 and Cdk6. FEBS Lett 587: 73-81, 2013.

13. Dou C, Wang Y, Li C, Liu Z, Jia Y, Li Q, Yang W, Yao Y, Liu Q and Tu K: MicroRNA-212 suppresses tumor growth of human hepatocellular carcinoma by targeting FOXA1. Oncotarget 6 : 13216-13228, 2015.

14. Ding G, Zhou L, Qian Y, Fu M, Chen J, Chen J, Xiang J, Wu Z, Jiang $G$ and Cao L: Pancreatic cancer-derived exosomes transfer miRNAs to dendritic cells and inhibit RFXAP expression via miR-212-3p. Oncotarget 6: 29877-29888, 2015.

15. Ramalinga M, Roy A, Srivastava A, Bhattarai A, Harish V, Suy S, Collins S and Kumar D: MicroRNA-212 negatively regulates starvation induced autophagy in prostate cancer cells by inhibiting SIRT1 and is a modulator of angiogenesis and cellular senescence. Oncotarget 6: 34446-34457, 2015.

16. Luo XJ, Tang DG, Gao TL, Zhang YL, Wang M, Quan ZX and Chen J: MicroRNA-212 inhibits osteosarcoma cells proliferation and invasion by down-regulation of Sox4. Cell Physiol Biochem 34: 2180-2188, 2014.
17. Hanieh H: Aryl hydrocarbon receptor-microRNA-212/132 axis in human breast cancer suppresses metastasis by targeting SOX 4 . Mol Cancer 14: 172, 2015

18. Li D, Li Z, Xiong J, Gong B, Zhang G, Cao C, Jie Z, Liu Y, Cao Y, Yan Y, et al: MicroRNA-212 functions as an epigenetic-silenced tumor suppressor involving in tumor metastasis and invasion of gastric cancer through down-regulating PXN expression. Am J Cancer Res 5: 2980-2997, 2015.

19. Liu H, Li C, Shen C, Yin F, Wang K, Liu Y, Zheng B, Zhang W, Hou X, Chen X, et al: MiR-212-3p inhibits glioblastoma cell proliferation by targeting SGK3. J Neurooncol 122: 431-439, 2015.

20. Ma C, Nong K, Wu B, Dong B, Bai Y, Zhu H, Wang W, Huang X, Yuan $\mathrm{Z}$ and Ai K: miR-212 promotes pancreatic cancer cell growth and invasion by targeting the hedgehog signaling pathway receptor patched-1. J Exp Clin Cancer Res 33: 54, 2014.

21. Qi B, Liu SG, Qin XG, Yao WJ, Lu JG, Guo L, Wang TY, Li HC and Zhao BS: Overregulation of microRNA-212 in the poor prognosis of esophageal cancer patients. Genet Mol Res 13: 7800-7807, 2014.

22. Li Y,Zhang D, Chen C, RuanZ,Li Y and Huang Y: MicroRNA-212 displays tumor-promoting properties in non-small cell lung cancer cells and targets the hedgehog pathway receptor $\mathrm{PTCH}$. Mol Biol Cell 23: 1423-1434, 2012.

23. Lu L, Zhang X, Zhang B, Wu J and Zhang X: Synaptic acetylcholinesterase targeted by microRNA-212 functions as a tumor suppressor in non-small cell lung cancer. Int J Biochem Cell Biol 45: 2530-2540, 2013.

24. Lamouille S, Xu J and Derynck R: Molecular mechanisms of epithelial-mesenchymal transition. Nat Rev Mol Cell Biol 15: 178-196, 2014.

25. Zhao JL, Zhang L, Guo X, Wang JH, Zhou W, Liu M, Li X and Tang H: miR-212/132 downregulates SMAD2 expression to suppress the G1/S phase transition of the cell cycle and the epithelial to mesenchymal transition in cervical cancer cells. IUBMB Life 67: 380-394, 2015.

26. Wada R, Akiyama Y, Hashimoto Y, Fukamachi H and Yuasa Y: miR-212 is downregulated and suppresses methyl-CpG-binding protein $\mathrm{MeCP} 2$ in human gastric cancer. Int $\mathbf{J}$ Cancer 127: 1106-1114, 2010

27. Hatakeyama H, Cheng $H$, Wirth P, Counsell A, Marcrom SR, Wood CB, Pohlmann PR, Gilbert J, Murphy B, Yarbrough WG, et al: Regulation of heparin-binding EGF-like growth factor by miR-212 and acquired cetuximab-resistance in head and neck squamous cell carcinoma. PLoS One 5: e12702, 2010.

28. Tu H, Wei G, Cai Q, Chen X, Sun Z, Cheng C, Zhang L, Feng Y, Zhou H, Zhou B, et al: MicroRNA-212 inhibits hepatocellular carcinoma cell proliferation and induces apoptosis by targeting FOXA1. Onco Targets Ther 8: 2227-2235, 2015.

29. Wu Z, Zhou L, Ding G and Cao L: Overexpressions of miR-212 are associated with poor prognosis of patients with pancreatic ductal adenocarcinoma. Cancer Biomark 18: 35-39, 2017.

30. Castillo SD, Matheu A, Mariani N, Carretero J, Lopez-Rios F, Lovell-Badge R and Sanchez-Cespedes M: Novel transcriptional targets of the SRY-HMG box transcription factor SOX4 link its expression to the development of small cell lung cancer. Cancer Res 72: 176-186, 2012.

31. Bilir B, Osunkoya AO, Wiles WG IV, Sannigrahi S, Lefebvre V, Metzger D, Spyropoulos DD, Martin WD and Moreno CS: SOX4 is essential for prostate tumorigenesis initiated by PTEN ablation. Cancer Res 76: 1112-1121, 2016. 